\title{
ON UNIQUENESS IN CAUCHY'S PROBLEM FOR ELLIPTIC OPERATORS WITH CHARACTERISTICS OF MULTIPLICITY GREATER THAN TWO ${ }^{1}$
}

\author{
BY MYRON M. SUSSMAN
}

Communicated by Alberto Calderón, December 31, 1974

The question of uniqueness in Cauchy's problem for elliptic partial differential operators has been reduced to the proof of certain integral estimates of Carleman type, viz.,

$$
\sigma \int|B(x, D) u(x)|^{2} e^{2 \tau \varphi_{p}(x)} d x \leqslant C \int|A(x, D) u(x)|^{2} e^{2 \tau \varphi_{p}(x)} d x
$$

or, in brief,

$$
\sigma\|B(x, D) u(x)\|^{2} \leqslant C\|A(x, D) u(x)\|^{2} \quad \forall u \in C_{0}^{\infty}(|x|<\delta / 2)
$$

where $x \in \mathbf{R}^{n}, \varphi_{p}=\left(x_{1}-\delta\right)^{2}+\delta^{p} \sum_{j=2}^{n} x_{j}^{2}, 1<p<2, \sigma \rightarrow \infty$ as $\delta \rightarrow 0$ or $\tau \longrightarrow \infty, C$ is a constant independent of the parameters $\delta, \tau$. Such an inequality is incompatible with the assumption that there is a solution $v(x)$ of the differential inequality $|A(x, D) v(x)| \leqslant C|B(x, D) v(x)|$ and an $\epsilon>0$ such that $v \equiv 0$ for $x_{1} \leqslant \epsilon \Sigma_{j=2}^{n} x_{j}^{2}$ unless there is a full neighborhood of $x=$ 0 on which $v \equiv 0$. Examples of such inequalities may be found in Hörmander [2], Pederson [3], Goorjian [1], and Watanabe [5], to mention only a few. The purpose of this note is to show how such inequalities may be obtained from simple assumptions involving the polynomial $A(x, \zeta)$.

We depart from custom and return to the classical notion of a multiindex $\alpha$ as a multiple of integers $\alpha=\left(\alpha_{1}, \ldots, \alpha_{k}\right), 1 \leqslant \alpha_{j} \leqslant n, j=1,2$, $\ldots, k$, and $|\alpha|=k$. We write $D_{j}=(1 / i)\left(\partial / \partial x_{j}\right), D^{j}=(1 / i)\left(\partial / \partial \zeta_{j}\right), D_{\alpha}=$ $D_{\alpha_{1}} D_{\alpha_{2}} \ldots D_{\alpha_{|\alpha|}}$ and $D^{\alpha}$ is defined similarly. We write $P^{(\alpha)}(x, \zeta)=$

AMS (MOS) subject classifications (1970). Primary 35J30, 35G10, 26 A84.

Key words and phrases. Elliptic partial differential operator, Cauchy problem, uniqueness, triple characteristics.

${ }^{1}$ This material is contained in the author's Ph.D. dissertation presented to the Department of Mathematics at Carnegie-Mellon University. The author wishes to thank his advisor, Professor R. N. Pederson for his direction and encouragement during its preparation. 
$D^{\alpha} P(x, \zeta)$ and $P_{(\alpha)}(x, \zeta)=D_{\alpha} P(x, \zeta)$. Write $\zeta=\left(\zeta_{1}, \zeta^{\prime}\right), \zeta^{\prime} \in \mathbf{C}^{n-1}$. If $P(x, \zeta)$ can be factored as a polynomial in $\zeta_{1}$ as

$$
P(x, \zeta)=\prod_{j=1}^{J}\left(\zeta_{1}-\rho_{j}\left(x, \zeta^{\prime}\right)\right)^{r_{j}}
$$

then we write the Lagrange interpolation polynomials

$$
\beta^{P(x, \zeta)}=\frac{P(x, \zeta)}{\left(\zeta_{1}-\rho_{\beta_{1}}\left(x, \zeta^{\prime}\right)\right)^{r_{1}} \ldots\left(\zeta_{1}-\rho_{\beta_{|\beta|}}\left(x, \zeta^{\prime}\right)\right)^{r_{\beta_{|\beta|}}}}
$$

where we restrict $\beta$ so that $1 \leqslant \beta_{j} \leqslant J$ and no entry $j$ is repeated more than $r_{j}$ times. If $F(\zeta)$ is differentiable, we write $\nabla F(\zeta) \in \mathbf{C}^{n}$ as the vector whose components are $D^{j} F(\zeta)$. If $V \subset \mathbf{R}^{n}$ is an open cone containing the vector $(-1,0, \ldots, 0)$ then we write $E(V)=\left\{\zeta \in \mathbf{C}^{n}: \zeta=\xi+i \tau N, \xi \in \mathbf{R}^{n}, \tau>0\right.$, $N \in V\}$. Finally, we use the letter $C$ to denote constants independent of parameters such as $p, \tau, \delta$, and the function $u$, and which may not be the same in different usages.

DEFInition. The homogeneous elliptic differential operator $A(x, D)$ is said to have nontangential characteristics of multiplicity $r$ at a point $\left(x_{0}, \zeta_{0}\right) \in \mathbf{R}^{n} \times \mathbf{C}^{n}$ if its symbol $A(x, \zeta)$ has the factorization in a neighborhood of $\left(x_{0}, \zeta_{0}\right)$

$$
A(x, \zeta)=\prod_{j=1}^{J}\left(\zeta_{1}-\rho_{j}\left(x, \zeta^{\prime}\right)\right)^{r_{j}}
$$

with $\rho_{j} \in C^{r-1}, j=1, \ldots, J$ where the $r_{j}$ are positive integers whose sum is $m, K \leqslant J$ is an integer such that $r_{1}+r_{2}+\ldots+r_{K}=r$ and

$$
\begin{aligned}
\rho_{1}\left(x_{0}, \zeta_{0}^{\prime}\right)=\rho_{2}\left(x_{0}, \zeta_{0}^{\prime}\right)=\ldots=\rho_{K}\left(x_{0}, \zeta_{0}^{\prime}\right) \neq & \rho_{j}\left(x_{0}, \zeta_{0}^{\prime}\right), \\
& j=K+1, \ldots, J,
\end{aligned}
$$

and the set of vectors $\left\{\gamma_{1}, \ldots, \gamma_{K}\right\} \subset \mathbf{C}^{n}$, is linearly independent, where $\gamma_{j}=\nabla\left(\zeta_{1}-\rho_{j}\right)$, evaluated at $\left(x_{0}, \zeta_{0}^{\prime}\right)$. We say that an operator has nontangential characteristics of multiplicity at most $r$ in a set if it has nontangential characteristics of multiplicity no greater than $r$ at every point in that set.

THEOREM. Suppose that $r$ is an odd integer, $V$ a cone, and either (a) $A(x, \zeta)=P(x, \zeta)$ is a homogeneous elliptic polynomial of degree $m$ whose roots with respect to $\zeta_{1}$ are locally $C^{r}$ in $E(V)$ and which are of multiplicity no greater than $r$; or 
(b) $A(x, \zeta)=P(x, \zeta)+Q(x, \zeta)$, where $P$ is homogeneous of degree $m$ and has nontangential characteristics of multiplicity no greater than $r$ in $E(V)$, and $Q$ is of degree $m-(r+1) / 2$ and has Lipschitz continuous coefficients.

Then there are constants $0<p<2,0<\delta_{0}, 0<\tau_{0}$ so that

$$
\sum_{|\alpha| \leqslant m}\left(\tau \delta^{2}\right)^{m-|\alpha|-r} \tau^{m-|\alpha|}\left\|D_{\alpha} u\right\|^{2} \leqslant C\|A(x, D) u(x)\|^{2}
$$

for all $0<\delta \leqslant \delta_{0}, \tau \geqslant \tau_{0} / \delta^{2}, u \in C_{0}^{\infty}(|x|<\delta / 2)$.

REMARK 1. Estimate (2) has the form of (1) when the summation is restricted to $|\alpha| \leqslant m-(r+1) / 2$.

REmarK 2. When $r=3$, (b) implies that the operator $(P(x, D)+$ $Q(x, D))+B(x, D)$, with $Q$ of degree $m-1$ and where $B$ is of degree no greater than $m-2$ and has bounded, measurable coefficients, satisfies uniqueness in Cauchy's problem.

REMark 3. When $P(x, D)=P_{1}(x, D) \circ P_{2}(x, D) \circ P_{3}(x, D)$ is the composition of homogeneous elliptic operators with simple characteristics and smooth coefficients, (b) is satisfied whenever $\left\{\nabla P_{1}(0, \zeta), \nabla P_{2}(0, \zeta), \nabla P_{3}(0, \zeta)\right\}$ is linearly independent for $\zeta \in E(V)$.

The proof of the Theorem is based on the inequality

$$
\left(\tau \delta^{2}\right)^{-|\alpha|}\left\|P_{(\alpha)}\left(x_{0}, D\right) u\right\|^{2} \leqslant C\left\|A\left(x_{0}, D\right) u\right\|^{2}
$$

for $1 \leqslant|\alpha| \leqslant r-1$, since the remainder of the proof is along lines used by Pederson [3] and Watanabe [5]. In the case (a), it is possible to derive (3) directly from the inequalities

$$
\left.P_{(\alpha)}(x, \zeta)\right|^{2} \leqslant\left.\left. C \sum_{|\beta|<|\alpha|}|\tau N|^{2}\right|_{\beta} P(x, \zeta)\right|^{2}, \quad 1 \leqslant|\alpha| \leqslant r-1,
$$

for $\zeta \in E(V)$ for some cone $V \subset \mathbf{R}^{n}$ containing $(-1,0, \ldots, 0)$. This inequality was first proved for $|\alpha|=1$ by Pederson [3]. In the case (b), (3) follows from the inequality

$$
\left|P_{(\alpha)}(x, \zeta)\right|^{2} \leqslant C \sum_{|\beta|<|\alpha|}|\tau N|^{2}\left|P^{(\beta)}(x, \zeta)\right|^{2}, \quad 1 \leqslant|\alpha| \leqslant r-1,
$$

for $\zeta \in E(V)$, and (5) can be shown by coupling (4) with the inequalities

$$
\text { (6) }\left.\left.\sum_{|\beta|=k}\right|_{\beta} P(x, \zeta)\right|^{2} \leqslant C \sum_{|\beta|=k}\left|P^{(\beta)}(x, \zeta)\right|^{2}, \quad k=1,2, \ldots,(r-1) \text {, }
$$

which are conscyuences of the nontangential assumption. The proof of (6) involves the consideration of many cases and will be published in full elsewhere. 


\section{REFERENCES}

1. P. M. Goorjian, The uniqueness of the Cauchy problem for partial differential equations which may have multiple characteristics, Trans. Amer. Math. Soc. 146 (1969), 493-509. MR 40 \#6048.

2. L. Hörmander, On the uniqueness of the Cauchy problem. II, Math. Scand. 7 (1959), 177-190. MR 22 \#12306.

3. R. N. Pederson, Uniqueness in Cauchy's problem for elliptic equations with double characteristics, Ark. Mat. 6 (1967), 535-549. MR 36 \#4111.

4. M. Sussman, On uniqueness in Cauchy's problem for elliptic partial differential operators of multiplicity greater than two, (to appear).

5. K. Watanabe, On the uniqueness of the Cauchy problem for certain elliptic equations with triple characteristics, Tôhoku Math. J. 23 (1971), 473-490. MR 46 \#7698.

DEPARTMENT OF MATHEMATICS, CARNEGIE-MELLON UNIVERSITY, PITTSBURGH, PENNSYLVANIA 15213 